DESY 14-092, HU-EP-14/25

June 2014

\title{
About the role of the Higgs boson in the evolution of the early universe *
}

\author{
Fred Jegerlehner,
}

\author{
Humboldt-Universität zu Berlin, Institut für Physik, \\ Newtonstrasse 15, D-12489 Berlin, Germany \\ Deutsches Elektronen-Synchrotron (DESY), \\ Platanenallee 6, D-15738 Zeuthen, Germany
}

\begin{abstract}
I review a recent analysis presented in [1,3]. After the discovery of the Higgs particle the most relevant structures of the SM have been verified and for the first time we know all parameters of the SM within remarkable accuracy. Together with recent calculations of the SM renormalization group coefficients up to three loops we can safely extrapolate running couplings high up in energy. Assuming that the SM is a low energy effective theory of a cutoff theory residing at the Planck scale, we are able to calculate the effective bare parameters of the underlying cutoff system. It turns out that the effective bare mass term changes sign not far below the Planck scale, which means that in the early universe the SM was in the symmetric phase. The sign-flip, which is a result of a conspiracy between the SM couplings and their screening/antiscreening behavior, triggers the Higgs mechanism. Above the Higgs phase transition the bare mass term in the Higgs potential must have had a large positive value, enhanced by the quadratic divergence of the bare Higgs mass. Likewise the quartically enhanced positive vacuum energy density is present in the symmetric phase. The Higgs system thus provides the large dark energy density in the early universe, which triggers slow-roll inflation, i.e. the SM Higgs is the inflaton scalar field. Reheating is dominated by the decay of the heavy Higgses into (in the symmetric phase) massless top/anti-top quark pairs. The Higgs mechanism stops inflation and the subsequent electroweak phase transition provides the masses to the SM particles in proportion to their coupling strength. The previously most abundantly produced particles are now the heaviest and decay into the lighter ones, by cascading down the CKM-element matrix from top and bottom to normal matter. Baryon-number $B$ violating interactions are naturally provided by Weinberg's set of close-by dimension 6 four-fermion effective interactions. Since matter is produced originating from the primordial heavy Higgs fields via $C$ and $C P$ violating decays we have actually a new scenario which possibly could explain the baryon-asymmetry essentially in terms of SM physics.
\end{abstract}

*Presented at the Cracow Epiphany Conference on physics at the LHC, Krakòw, Poland, January 8-10, 2014. 


\section{Introduction}

With the discovery of the Higgs boson by ATLAS [4] and CMS [5] at the LHC all relevant ingredients of the Standard Model (SM) have been established experimentally. In particular, for the first time we know all the basic SM parameters with remarkable accuracy. The Higgs mass, found to be $M_{H}=125.9 \pm 0.4 \mathrm{GeV}$, turned out to have a value just in the window which was required to allow one to extrapolate SM physics up to the Planck scale [6] without the need to assume some new non-SM physics. This together with the fact that so far no hints for a supersymmetric extension or extra dimensions etc. have been found, sheds new light on the structure of the SM and its self-consistency. The SM together with its specific values for the couplings, the gauge couplings $g^{\prime}, g, g_{s}$, the top-quark Yukawa coupling $y_{t}$ and newly, the Higgs self-coupling $\lambda$ are supporting the picture of the SM as a low energy effective theory of some cutoff system residing at the Planck scale. In such a framework the relation between bare and renormalized physical low energy parameters acquirers a physical meaning and from the knowledge of the physical parameters we can calculate actually the bare parameters relevant at the high (short distance) scale. The SM as a low energy theory, is then emerging as a result of the low energy expansion in $E / \Lambda_{\mathrm{Pl}}$. All positive powers $\left(E / \Lambda_{\mathrm{Pl}}\right)^{n}, n=1,2,3, \cdots$ are heavily suppressed by the very high cutoff $\Lambda_{\mathrm{Pl}} \sim 10^{19} \mathrm{GeV}$ and unobservable at present accelerator energies. Renormalizability of the SM as well as all known conditions which where required to get the SM as a minimal renormalizable extension of its low energy effective structure now are a consequence of the low energy expansion. As we do not see the infinite tower of non-renormalizable effective operators, the low energy effective theory actually has more symmetry than the underlying cutoff system at the Planck scale, which is largely unknown in its details. In such a scenario simplicity and symmetries are expected to be naturally generated dynamically as a consequence of our blindness for the details of the underlying cutoff system.

Our scenario, not new at all, has to be seen in the context of the general question about "What is the path to physics at the Planck scale?". The String Paradigm assumes that "the closer we look the more symmetric the world looks like" assuming a hierarchy of symmetries like

$$
\mathrm{M}-\text { Theory } \sim \text { Strings } \leftarrow \text { SUGRA } \leftarrow \text { SUSY } \leftarrow \text { SM }
$$

In contrast the Emergence Paradigm understands nature as "the less close you look the simpler it looks"

$$
\text { Planck medium "Ether" } \rightarrow \text { low energy effective QFT } \rightarrow \text { SM. }
$$

The latter view understands the SM as the "true world" seen from far away. The methodological approach we know from investigating the long range properties of condensed matter systems, specifically, critical phenomena, which may be applied to particle physics as well. In this context even the quantum field theories are structures emergent from critical and quasi critical underlying condensed matter systems.

At the high scale, given by the intrinsic cutoff, one expects all kinds of excitations. Most of them cannot be seen at long distances (non-critical modes), however, conspiracies between 
modes are able to develop quasi critical modes which are seen as light particles in interaction, which take the form of a non-trivial renormalizable QFT in space-time of dimension $D=4$. For $D>4$ only trivial stable theories would exist, such that extra dimensions decouple. About the details of the "ether" we do not know much, except that we have to stay within the universality class (三 the totality of possible systems exhibiting identical long range tail) of the SM. Such a view turns upside-down the standard believes that the higher the energy the simpler the world, together with the assumption that symmetries in nature are broken at best spontaneously. Such scenarios assume renormalizability as a basic principle and symmetries to be broken by the relevant operators of dimension $d<4$ and ignore the fact that there is a infinite tower of possible higher order operators with $d>4$, which generally would violate symmetries seen at low energy.

Symmetries relevant for the SM are small gauge groups, with particles in multiplets of few conspiring fields, like doublets and triplets, i.e. the SM gauge structure is natural in a low energy expansion. In contrast, GUT symmetries are not naturally emergent and have to be put by hand at the high scale.

In this scenario the relation between bare and renormalized parameters is physical and bare parameters predictable from known renormalized ones. All so called "UV singularities" must be taken serious including terms enhanced quadratically and quartically in the cutoff. Since the cutoff is finite there are no divergences and a cutoff limit is not required to exist. The impact of the very high Planck cutoff is that the local renormalizable QFT structure of the SM is presumably valid up not far below the Planck scale. This also justifies the application of the SM RG up to high scales.

\section{Low energy effective QFT of a cutoff system}

I think it is instructive to be more specific about the appearance of low energy effective theories. The best would be to implement the SM as a lattice field theory in the unitary gauge, in which the Higgs vacuum expectation value (VEV) $v$ is a well defined order parameter of the broken $H \leftrightarrow-H\left(Z_{2}\right)$ symmetry, with a small lattice spacing $a=\Lambda^{-1}$, and take the lattice system as the true underlying theory and work out its long range properties. In order to illustrate the emergence of a low energy effective theory, for simplicity, let us consider the cutoff version of a self-interacting Higgs system with Lagrangian (for details see [7])

$$
\mathcal{L}=\mathcal{L}_{0}+\mathcal{L}_{\text {int }}=\frac{1}{2} \partial^{\mu} \phi(x)\left(1+\square / \Lambda^{2}\right) \partial_{\mu} \phi(x)-\frac{1}{2} m_{0}^{2} \phi(x)^{2}-\frac{\lambda_{0}}{4 !} \phi^{4}(x) .
$$

The regularization is chosen here as a Pais-Uhlenbeck higher-derivative kinetic cutoff term.

We consider a vertex function (connected amputated one-particle irreducible diagrams) of $N$ scalar fields. The bare vertex functions are related to the renormalized ones by reparametrizing parameters and fields

$$
\Gamma_{\Lambda r}^{(N)}(p ; m, \lambda)=Z^{N / 2}(\Lambda / m, \lambda) \Gamma_{\Lambda b}^{(N)}\left(p ; \Delta m_{0}(\Lambda, m, \lambda), \lambda_{0}(\Lambda / m, \lambda)\right) .
$$

The renormalized functions satisfy a RG equation which controls the response to a change

of the cutoff $\Lambda:\left.\Lambda \frac{\partial}{\partial \Lambda} \Gamma(N)_{\Lambda b}\right|_{m, \lambda}$, for fixed renormalized parameters, and which by applying 
the chain rule of differentiation yields

$$
\begin{aligned}
& \left(\Lambda \frac{\partial}{\partial \Lambda}+\beta_{0} \frac{\partial}{\partial \lambda}-N \gamma_{0}+\delta_{0} \Delta m_{0}^{2} \frac{\partial}{\partial \Delta m_{0}^{2}}\right) \Gamma_{\Lambda b}^{(N)}\left(p ; m_{0}, \lambda_{0}\right) \\
= & Z^{-N / 2} \Lambda \frac{\partial}{\partial \Lambda} \Gamma_{\Lambda r}^{(N)}(p ; m, \lambda) .
\end{aligned}
$$

$m_{0 c}^{2}$ is the "critical value" of the bare mass for which the renormalized mass is zero, i.e. $\left.\Gamma_{\Lambda b}^{(2)}\right|_{p=0}=0$, and $\Delta m_{0}^{2}=m_{0}^{2}-m_{0 c}^{2}$ corresponds to the renormalized mass parameter. Since the renormalized vertex functions have a regular limit as $\Lambda \rightarrow \infty$, to all orders in perturbation theory the inhomogeneous part behaves as

$$
Z^{N / 2} \Lambda \frac{\partial}{\partial \Lambda} \Gamma_{\Lambda r}^{(N)}(p ; m, \lambda)=O\left(\Lambda^{-2}(\ln \Lambda)^{l}\right),
$$

i.e., the inhomogeneous part, representing a cutoff insertion, falls off faster than the l.h.s. of Eq. (3) by two powers in the cutoff for large cutoffs. This is easy to understand given the fact that the cutoff enters $\mathcal{L}$ as a term proportional to $\Lambda^{-2}$. All the RG equation coefficients exist as non-trivial functions in the limit of infinite cutoff:

$$
\lim _{\Lambda \rightarrow \infty} \alpha_{0}(\Lambda / m, \lambda)=\alpha(\lambda), \alpha=\beta, \gamma, \delta,
$$

for dimensions $2 \leq D \leq 4$. In $D=4$ dimensions the proper vertex-functions have a large cutoff $\Lambda$-expansion

$$
\Gamma_{\Lambda b}^{(N)}\left(p ; \Delta m_{0}, \lambda_{0}\right)=\sum_{k, l \geq 0} \Lambda^{-2 k}(\ln \Lambda)^{l} f_{k l}^{(N)}\left(p \Delta m_{0}, \lambda_{0}\right)
$$

and for large $\Lambda$ we obtain the preasymptote of $\Gamma_{\Lambda b}^{(N)}$

$$
\Gamma_{\Lambda \text { as }}^{(N)}\left(p ; \Delta m_{0}, \lambda_{0}\right)=\sum_{l \geq 0}(\ln \Lambda)^{l} f_{0 l}^{(N)}\left(p \Delta m_{0}, \lambda_{0} \Lambda^{\varepsilon}\right),
$$

which is collecting all leading terms and satisfies the bound

$$
\left|\Gamma_{\Lambda b}^{(N)}\left(p ; \Delta m_{0}, \lambda_{0}\right)-\Gamma_{\Lambda \text { as }}^{(N)}\left(p ; \Delta m_{0}, \lambda_{0}\right)\right|=O\left(\Lambda^{-2}\left(\ln \Lambda^{l_{x}}\right)\right) .
$$

The index $l_{x}$ is bounded to all orders in the perturbation expansion. The key point is that the still cutoff dependent preasymptote satisfies a homogeneous RG equation, a special property of the long range tail of the bare theory:

$$
\begin{aligned}
& \left(\Lambda \frac{\partial}{\partial \Lambda}+\beta_{\mathrm{as}}\left(\Lambda / \Delta m_{0}, \lambda_{0}\right) \frac{\partial}{\partial \lambda_{0}}-N \gamma_{\mathrm{as}}\left(\Lambda / \Delta m_{0}, \lambda_{0}\right)\right. \\
& \left.\quad+\delta_{\mathrm{as}}\left(\Lambda / \Delta m_{0}, \lambda_{0}\right) \Delta m_{0}^{2} \frac{\partial}{\partial \Delta m_{0}^{2}}\right) \Gamma_{\Lambda \text { as }}^{(N)}\left(p ; \Delta m_{0}, \lambda_{0}\right)=0
\end{aligned}
$$

The homogeneity of this partial differential equation for the response to a change in $\Lambda$ means that $\Lambda$ does not represent a cutoff any more and just takes the role of a renormalization scale parameter. The interpretation (verifiable to all orders in the perturbation expansion) is the following: 
- the preasymptotic theory is a non-trivial local relativistic QFT;

- the crucial point is that the cutoff $\Lambda$ is physical i.e. a finite number and by a finite renormalization (renormalizing parameters and fields only) by change of scale $p_{i} \rightarrow$ $\kappa p_{i} ; \kappa=\Lambda / \mu$ one can achieve that momenta measured in units of $\Lambda$ are rescaled to momenta expressed in units of $\overline{\mathrm{MS}}$ scale $\mu$;

- as a consequence, the relationship between renormalized and bare parameters is physical, such that knowing the renormalized parameters we are able to calculate the bare ones;

- an important empirical fact: besides QCD at low energy, elementary particle interactions have rather weak coupling such that perturbation theory works in general;

- applied to our "real world" physics with $\Lambda=\Lambda_{\mathrm{Pl}}$ the cutoff is very very high such that all cutoff structure are deeply hidden at present accelerator energies.

A comparison with QCD reveals the importance of a large cutoff. Low energy effective hadron theories suffer from the close-by cutoff and are therefore difficult to establish unambiguously. In Table 1 we give another representation of the low energy expansion at work:

Table 1: Typical operators in a low energy expansion

\begin{tabular}{|c|c|c|c|}
\hline & $\begin{array}{l}\text { dimension } \\
\end{array}$ & operator & scaling behavior \\
\hline $\begin{array}{c}\uparrow \\
\text { no } \\
\text { data } \\
\mid\end{array}$ & $\begin{array}{l}d=6 \\
d=5\end{array}$ & $\begin{array}{c}\infty-\text { many } \\
\text { irrelevant } \\
\text { operators } \\
(\square \phi)^{2},(\bar{\psi} \psi)^{2}, \cdots \\
\bar{\psi} \sigma^{\mu \nu} F_{\mu \nu} \psi, \cdots\end{array}$ & $\begin{array}{c}\left(E / \Lambda_{\mathrm{Pl}}\right)^{2} \\
\left(E / \Lambda_{\mathrm{Pl}}\right)\end{array}$ \\
\hline \multirow{2}{*}{$\begin{array}{c}\mid \\
\text { experimental } \\
\text { data } \\
\downarrow\end{array}$} & $d=4$ & $(\partial \phi)^{2}, \phi^{4},\left(F_{\mu \nu}\right)^{2}$ & $\ln \left(E / \Lambda_{\mathrm{Pl}}\right)$ \\
\hline & $\begin{array}{l}d=3 \\
d=2 \\
d=1\end{array}$ & $\begin{array}{c}\phi^{3}, \bar{\psi} \psi \\
\phi^{2},\left(A_{\mu}\right)^{2} \\
\phi\end{array}$ & $\begin{array}{c}\left(\Lambda_{\mathrm{Pl}} / E\right) \\
\left(\Lambda_{\mathrm{Pl}} / E\right)^{2} \\
\left(\Lambda_{\mathrm{Pl}} / E\right)^{3}\end{array}$ \\
\hline
\end{tabular}

The relevant operators must be tamed by symmetries, in order not to blow up with the cutoff: chiral symmetry and gauge symmetry in the SM, and supersymmetry in supersymmetric extensions of the SM. 
Up to date and for a long time to come there is and will be no direct experimental information on $O\left(E / \Lambda_{\mathrm{Pl}}\right)$ or $O\left(E^{2} / \Lambda_{\mathrm{Pl}}^{2}\right)$ effects, but bounds on the absence of such terms, unless they violate basic SM symmetries like baryon-number conservation, for example.

The infinite tower of irrelevant operators of dimension $>4$ are not seen at low energy and imply the simplicity of the SM! Blindness to details implies more symmetries (YangMills structure [gauge cancellations] with small groups 1 : doublets, triplets besides singlets, Lorentz invariance, anomaly cancellation and family structure, triviality for space-time dimensions $D>4[\mathrm{D}=4$ border case for an interacting world at long distances, this has nothing to do with compactification, extra dimensions just trivialize by themselves], etc.). The natural emergence of spin 1 and spin 2 excitations has been considered in Ref. [8].

Problems are posing the relevant operators of dimension $<4$. In particular the mass terms, require "tuning to criticality". In the symmetric phase of the SM we are confronted with one mass term only (the others are forbidden by the known chiral and gauge symmetries), the one of the Higgs doublet field.

The symmetric phase Higgs fine tuning has the form

$$
m_{0}^{2}=m^{2}+\delta m^{2} ; \delta m^{2}=\frac{\Lambda^{2}}{32 \pi^{2}} C,
$$

with a coefficient typically $C=O(1)$. To keep the renormalized mass at some small value, which can be seen at low energy, $m_{0}^{2}$ has to be adjusted to compensate the huge number $\delta m^{2}$ such that about 35 digits must be adjusted in order to get the observed value around the electroweak scale. This is the usual hierarchy problem.

\section{Matching and running couplings}

The key questions asked here are: 1) how does SM physics look like at much higher energies and 2) what does the Higgs potential look like at the bare level, when going to the Planck scale. The first question can be answered, under the assumption that no substantial effects come in by possible physics beyond the SM, by studying the evolution of couplings as determined by the SM renormalization group (RG), which now is known to three loops in the $\overline{\mathrm{MS}}$ renormalization scheme [10 15]. The initial $\overline{\mathrm{MS}}$ values have to be obtained by appropriate matching conditions from the physical on-shell parameters. For the latter we use the values [21]:

$$
\begin{aligned}
& M_{Z}=91.1876(21) \mathrm{GeV}, \quad M_{W}=80.385(15) \mathrm{GeV}, \quad M_{t}=173.5(1.0) \mathrm{GeV}, \\
& G_{\mu}=1.16637(1) \times 10^{-5} \mathrm{GeV}^{-2}, \quad \hat{G}_{\mu}=G_{\mu}\left(M_{Z}\right)=1.15564(55) \times 10^{-5} \mathrm{GeV}^{-2} \\
& \alpha^{-1}=137.035999, \quad \alpha^{-1}\left(M_{Z}^{2}\right)=127.944, \quad \alpha_{s}\left(M_{Z}^{2}\right)=0.1184(7) .
\end{aligned}
$$

For the Higgs mass we adopt

$$
M_{H}=125.9 \pm 0.4 \mathrm{GeV},
$$

\footnotetext{
${ }^{1}$ Such a pattern (few particle multiplets) reminds of primordial nucleosynthesis, which exclusively produces only the simplest, i.e. lightest, elements.
} 
in accord with latest ATLAS and CMS reports. All light-fermion masses $M_{f}(f \neq t)$ give negligible effects and do not play any role in our consideration. The top quark mass given above is taken to be the pole mass. It should be reminded that it is not precisely clear whether the value reported by experiments or by the PDG can be identified with the on-shell mass within the given accuracy. For a recent review on the subtleties in defining/measuring the top quark mass see e.g. [22] and references therein.

One somewhat controversial issue about the electroweak matching conditions concern the inclusion of tadpole contributions in the relationship between on-shell and $\overline{\mathrm{MS}}$ quantities. The tadpoles which only show up in the broken phase, where they results from the radiative corrections of the Higgs VEV $v$, on the one hand can yield large corrections, on the other hand there is a theorem which says that tadpole contributions drop out from relations between measured quantities. For this reason tadpoles are often dropped in actual calculations. It should be realized that experimentally measured quantities incorporate tadpole contributions in any case (one cannot exclude subsets of diagrams form a measurement). The relation between $\overline{\mathrm{MS}}$ and on-shell quantities, however is not a relation between physical quantities and tadpoles are relevant to be included. Since tadpoles are neither gauge-invariant nor UV finite, dropping them leads to gauge dependent quasi- $\overline{\mathrm{MS}}$ parameters which in addition do not satisfy the correct RG equations (see [24, 25, 29, 30]). Care has to be taken also of the fact that the weak corrections are not respecting the Appelquist-Carazzone theorem [26] when evaluating the matching conditions. This means for example that electroweak top quark contributions do not start above the top quark threshold. Top quarks e.g. give a large contribution to the $\rho$-parameter

$$
\begin{aligned}
\rho(0) \equiv & G_{\mathrm{NC}}(0) / G_{\mu}(0)=1+\frac{3 \sqrt{2} G_{\mu}}{16 \pi^{2}}\left\{M_{t}^{2}\right. \\
& \left.+\left(\frac{M_{W}^{2}}{1-M_{W}^{2} / M_{H}^{2}} \ln M_{H}^{2} / M_{W}^{2}-\frac{M_{Z}^{2}}{1-M_{Z}^{2} / M_{H}^{2}} \ln M_{H}^{2} / M_{Z}^{2}+\cdots\right)\right\},
\end{aligned}
$$

where $G_{\mathrm{NC}}(0)$ and $G_{\mu}(0)$ are the neutral and charged current effective Fermi couplings at zero momentum, respectively.

The top Yukawa coupling and the Higgs self-coupling are only known via their measured masses via the mass coupling relations

$$
\begin{aligned}
m_{W}^{2}\left(\mu^{2}\right) & =\frac{1}{4} g^{2}\left(\mu^{2}\right) v^{2}\left(\mu^{2}\right) ; m_{Z}^{2}\left(\mu^{2}\right)=\frac{1}{4}\left(g^{2}\left(\mu^{2}\right)+g^{2}\left(\mu^{2}\right)\right) v^{2}\left(\mu^{2}\right) ; \\
m_{f}^{2}\left(\mu^{2}\right) & =\frac{1}{2} y_{f}^{2}\left(\mu^{2}\right) v^{2}\left(\mu^{2}\right) ; m_{H}^{2}\left(\mu^{2}\right)=\frac{1}{3} \lambda\left(\mu^{2}\right) v^{2}\left(\mu^{2}\right),
\end{aligned}
$$

which derive from the Higgs mechanism.

We will have to distinguish bare and renormalized quantities and among the latter $\overline{\mathrm{MS}}$ and physical on-shell ones. As usual we adopt dimensional renormalization starting form $D=4-\varepsilon$ dimensions and taking the limit $\varepsilon \rightarrow+0$ after renormalization. By $m_{i 0}$ we denoted the bare, by $m_{i}$ the $\overline{\mathrm{MS}}$ and by $M_{i}$ the on-shell masses. $\operatorname{Reg}=\frac{2}{\varepsilon}-\gamma+\ln 4 \pi+\ln \mu_{0}^{2}$ is the UV regulator term with $\mu_{0}$ the bare scale parameters used in dimensional renormalization. The 
substitution Reg $\rightarrow \ln \mu^{2}$ defines the UV finite $\overline{\mathrm{MS}}$ parametrization. Let $\delta M_{b}^{2}$ denote the bare on-shell mass counterterm for a boson species $b$ and $\delta M_{f}$ the corresponding counterterm for a fermion species $f$. By identifying $m_{b}^{2}\left(\mu^{2}\right)=M_{b}^{2}+\left.\delta M_{b}^{2}\right|_{\operatorname{Reg}=\ln \mu^{2}}$ and $m_{f}\left(\mu^{2}\right)=M_{f}+$ $\left.\delta M_{f}\right|_{\operatorname{Reg}=\ln \mu^{2}}$, respectively, we then obtain the $\overline{\mathrm{MS}}$ masses in terms of the on-shell masses. Similar relations apply for the coupling constants $g, g^{\prime}, \lambda$ and $y_{f}$, which, however, usually are fixed using the mass-coupling relations in terms of the masses and the Higgs VEV, which is determined by the Fermi constant as $v=\left(\sqrt{2} G_{\mu}\right)^{-1 / 2}$. Here $G_{\mu}$ is the muon decay constant, which represents the Fermi constant in the on-shell scheme. The $\overline{\mathrm{MS}}$ version of the Fermi constant we denote by $G_{F}^{\overline{\mathrm{MS}}}$ or simply by $G_{F}$. The matching condition for the Higgs VEV may be represented in terms of the one for the muon decay constant

$$
G_{F}^{\overline{\mathrm{MS}}}\left(\mu^{2}\right)=G_{\mu}+\left(\left.\delta G_{\mu}\right|_{\mathrm{OS}}\right)_{\mathrm{Reg}=\ln \mu^{2}},
$$

where $\left.\frac{\delta G_{\mu}}{G_{\mu}}\right|_{\mathrm{OS}}=2 \frac{\delta v^{-1}}{v^{-1}}$. for details I refer to Ref. [24, 25]. Then the $\overline{\mathrm{MS}}$ top quark Yukawa coupling is given by

$$
y_{t}^{\overline{\mathrm{MS}}}\left(M_{t}^{2}\right)=\sqrt{2} \frac{m_{t}\left(M_{t}^{2}\right)}{v^{\overline{\mathrm{MS}}}\left(M_{t}^{2}\right)} ; v^{\overline{\mathrm{MS}}}\left(\mu^{2}\right)=\left(\sqrt{2} G_{F}^{\overline{\mathrm{MS}}}\right)^{-1 / 2}\left(\mu^{2}\right)
$$

and the other $\overline{\mathrm{MS}}$ mass-coupling relations correspondingly. The RG equation for $v^{2}\left(\mu^{2}\right)$ follows from the RG equations for masses and coupling of the Higgs potential $V(\phi)=$ $\frac{1}{2} m^{2} \phi^{2}+\frac{1}{24} \lambda \phi^{4}$ as

$$
v^{2}\left(\mu^{2}\right)=3 \frac{m_{H}^{2}\left(\mu^{2}\right)}{\lambda\left(\mu^{2}\right)} ; \mu^{2} \frac{d}{d \mu^{2}} v^{2}\left(\mu^{2}\right)=v^{2}\left(\mu^{2}\right)\left[\gamma_{m^{2}}-\frac{\beta_{\lambda}}{\lambda}\right] .
$$

We remind that all dimensionless couplings satisfy the same RG equations in the broken and in the unbroken phase. Figure 1 shows the solutions of the RG equations and the $\beta$-functions up to $\mu=M_{\text {Planck. }}$.

Remarkably, as previously found for the running couplings in Refs. [16-20], all parameters stay in bounded ranges up to the Planck scale if one adopts our matching conditions together with the so far calculated RG coefficients. We note that including all known terms no transition to a metastable state in the effective Higgs potential is observed with our set of $\overline{\mathrm{MS}}$ input parameters, i.e. no change of sign in $\lambda$ occurs, in agreement with Refs. [16, 18]. Results at various scales are collected in Table 2 .

\section{The quadratic divergences in the SM}

In the unbroken phase the only quadratic divergences show up in the renormalization of Higgs potential mass $m$. Since the UV structure is the same in the broken phase, there are no other problems in this direction. Here we encounter the fine tuning relation (10). At one-loop the coefficient function $C_{1}$ has been discussed within this context by Veltman [23], and modulo small lighter fermion contributions is given by

$$
C_{1}=\frac{6}{v^{2}}\left(M_{H}^{2}+M_{Z}^{2}+2 M_{W}^{2}-4 M_{t}^{2}\right)=2 \lambda+\frac{3}{2} g^{\prime 2}+\frac{9}{2} g^{2}-12 y_{t}^{2} .
$$



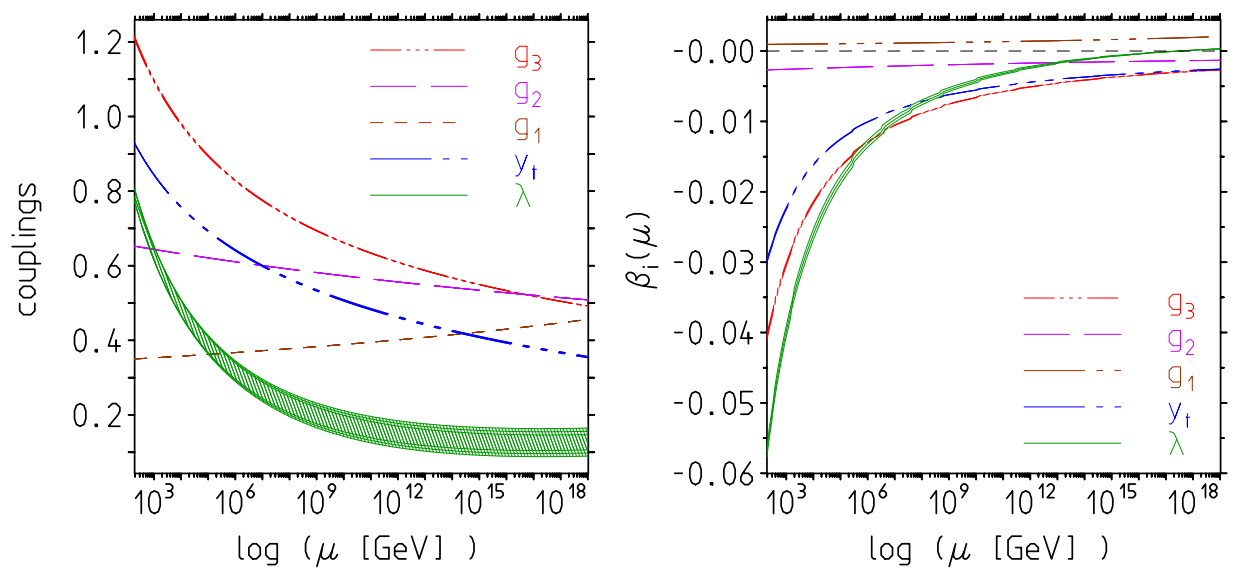

Figure 1: Left: the dimensionless SM couplings in the $\overline{\mathrm{MS}}$ scheme as a function of the renormalization scale (see also [16 20]). The input parameter uncertainties as given in RPP [21] are represented by the thickness of the lines. The gray/green band corresponds to Higgs masses in the range [124-127] GeV. Right: the $\beta$-functions for the couplings $g_{3}, g_{2}, g_{1}$, $y_{t}$ and $\lambda$. The uncertainties are represented by the line widths.

On the one hand parameters are known in the broken low energy phase, where they are directly accessible to experiment, on the other hand they are given in terms of SM parameters in the unbroken phase, which is physical at high energies. A priori, the renormalized $m^{2}$ in the symmetric phase is not known and not accessible directly to experiment. As we will see below, if $m^{2}$ would not be small relative to the very large $\delta m^{2}$ it would affect the inflation pattern and thus in principle is constrained by the observed Cosmic Microwave Background (CMB) fluctuation data. In fact the matching condition $m_{0}^{2}=m^{2}$ at scale $\mu_{0}$ where $\delta m^{2}=0$ actually fixes the renormalized mass at any scale in terms of the measured Higgs mass and the RG evolution of it. So the hierarchy problem seems to be a problem in the symmetric phase. In order to understand this we have to be aware that the Higgs is not a fundamental mode in the underlying cutoff system. Therefore, in the underlying cutoff system $m_{0}^{2}$ is not a fundamental parameter, but an effective one associated with the scalar Higgs mode, which usually is some collective effect within the Planck medium. This means that the effective bare mass is actually essentially generated by the dynamics and hence largely determined by $\delta m^{2}$, i.e. the bare mass is radiatively generated. In any case we assume $m^{2}$ to be small relative to $\delta m^{2}$.

What is important is that $C_{1}$ is universal and depends on dimensionless gauge, Yukawa and Higgs self-coupling only, the RGs of which are unambiguous. Similarly, for the two-loop coefficient $C_{2}$, first calculated in Refs. [27,28,

$$
\begin{aligned}
C_{2}= & C_{1}+\frac{\ln \left(2^{6} / 3^{3}\right)}{16 \pi^{2}}\left[18 y_{t}^{4}+y_{t}^{2}\left(-\frac{7}{6} g^{\prime 2}+\frac{9}{2} g^{2}-32 g_{s}^{2}\right)\right. \\
& \left.-\frac{87}{8} g^{\prime 4}-\frac{63}{8} g^{4}-\frac{15}{4} g^{2} g^{\prime 2}+\lambda\left(-6 y_{t}^{2}+g^{\prime 2}+3 g^{2}\right)-\frac{2}{3} \lambda^{2}\right],
\end{aligned}
$$

which numerically does not change significantly the one-loop result. Recently, Hamada, 
Table 2: Parameters in $\overline{\mathrm{MS}}$ scheme at various scales for $M_{H}=126 \mathrm{GeV}$ and $\mu_{0} \simeq 1.4 \times$ $10^{16} \mathrm{GeV} . C_{1}$ and $C_{2}$ are the one- and two-loop coefficients of the quadratic divergence, respectively. $C_{1}$ given by Eq. (17). The last two columns show corresponding results from Ref. [20].

\begin{tabular}{ccccc||cr}
\hline coupling \scale & $M_{Z}$ & $M_{t}$ & $\mu_{0}$ & $M_{\mathrm{Pl}}$ & $M_{t}[20]$ & $M_{\mathrm{Pl}}[20]$ \\
\hline$g_{3}$ & 1.2200 & 1.1644 & 0.5271 & 0.4886 & 1.1644 & 0.4873 \\
$g_{2}$ & 0.6530 & 0.6496 & 0.5249 & 0.5068 & 0.6483 & 0.5057 \\
$g_{1}$ & 0.3497 & 0.3509 & 0.4333 & 0.4589 & 0.3587 & 0.4777 \\
$y_{t}$ & 0.9347 & 0.9002 & 0.3872 & 0.3510 & 0.9399 & 0.3823 \\
$y_{b}$ & 0.0238 & 0.0227 & 0.0082 & 0.0074 & & \\
$y_{\tau}$ & 0.0104 & 0.0104 & 0.0097 & 0.0094 & & \\
$\sqrt{\lambda}$ & 0.8983 & 0.8586 & 0.3732 & 0.3749 & 0.8733 & i 0.1131 \\
$\lambda$ & 0.8070 & 0.7373 & 0.1393 & 0.1405 & 0.7626 & -0.0128 \\
$C_{1}$ & -6.768 & -6.110 & 0 & 0.2741 & & \\
$C_{2}$ & -6.672 & -6.217 & 0 & 0.2845 & & \\
$m[\mathrm{GeV}]$ & 89.096 & 89.889 & 97.278 & 96.498 & 97.278 & \\
\hline
\end{tabular}

Kawai and Oda [19] have investigated the coefficients to two loops in terms of running couplings and found the coefficients of the quadratic divergence to have a zero not far above the Planck scale. For the parameters listed in Table 2, the SM makes a prediction for the coefficients $C_{i}$ and hence for the bare mass parameter in the Higgs potential, which we displayed in Fig. 2, In the broken phase given by $m_{0}^{2}=\frac{1}{2} m_{H 0}^{2}, m_{0}^{2}$ is calculable and is exhibiting the following properties: i) the coefficient $C_{n}(\mu)$ exhibits a zero, for $M_{H}=$ $126 \mathrm{GeV}$ at about $\mu_{0} \sim 1.4 \times 10^{16}$, not far below $\mu=M_{\text {Planck }}$, ii) at the zero of the coefficient function the counterterm $\delta m^{2}=m_{0}^{2}-m^{2}=0$ vanishes and the bare mass changes sign, iii) this represents a first order phase transition which triggers the Higgs mechanism and seems to play an important role for cosmic inflation, iv) at the transition point $\mu_{0}$ we have $v_{0}=v\left(\mu_{0}^{2}\right)$, where $v\left(\mu^{2}\right)$ is the $\overline{\mathrm{MS}}$ renormalized Higgs VEV, v) the jump in the vacuum density, thus agrees with the renormalized one: $-\Delta \rho_{\text {vac }}=\frac{\lambda\left(\mu_{0}^{2}\right)}{24} v^{4}\left(\mu_{0}^{2}\right)$, and thus is $O\left(v^{4}\right)$ and not $O\left(M_{\text {Planck }}^{4}\right)$.

We note that $\beta_{\lambda}$ has a zero at about $\mu_{\lambda} \sim 3.5 \times 10^{17}>\mu_{0}$, where the Higgs self-coupling $\lambda$ although rather small is still positive and then starts slowly increasing up to $M_{\text {Planck }}$ [24].

In any case the zero of the coefficient function $C(\mu)$ triggers a phase transition, which corresponds to a restoration of the symmetry. Indeed, there is a close relation between the Higgs mechanism and the electroweak (EW) phase transition [31]. To this end we have to consider the relevant finite temperature effects [32 34], which are dominating especially in the very early thermal evolution of the universe at the hot big bang. Including the leading effect only, the finite temperature effective potential reads

$$
V(\phi, T)=\frac{1}{2}\left(g_{T} T^{2}-\mu^{2}\right) \phi^{2}+\frac{\lambda}{24} \phi^{4}+\cdots .
$$



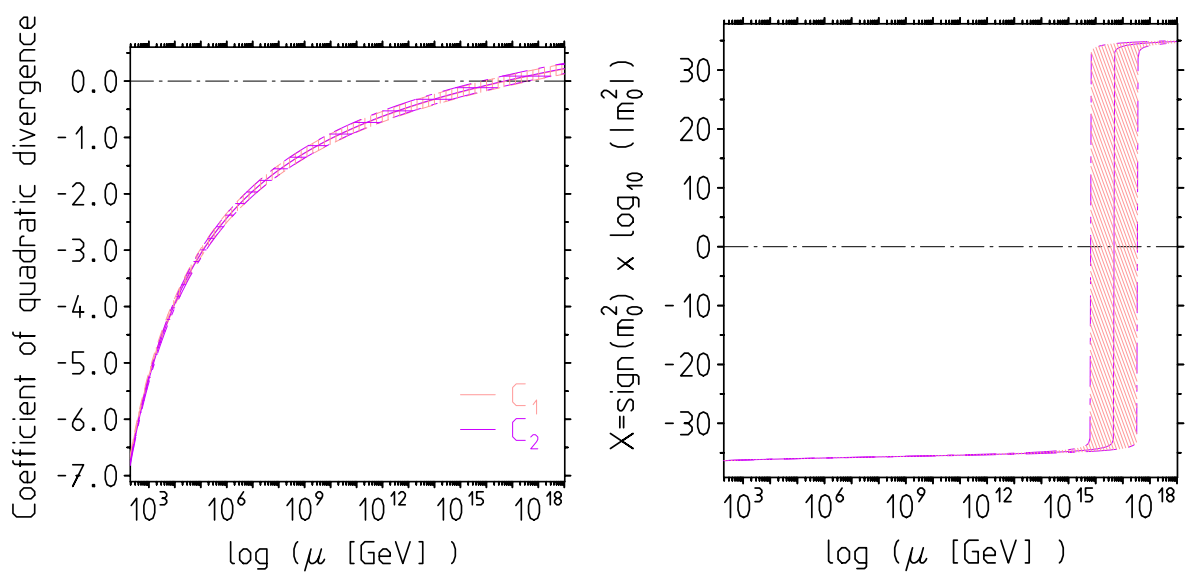

Figure 2: The Higgs phase transition in the SM. Left: the zero in $C_{1}$ and $C_{2}$ for $M_{H}=125.9 \pm$ $0.4 \mathrm{GeV}$. Right: shown is $X=\operatorname{sign}\left(m_{0}^{2}\right) \times \log _{10}\left(\left|m_{0}^{2}\right|\right)$, which represents $m_{0}^{2}=\operatorname{sign}\left(m_{0}^{2}\right) \times 10^{X}$.

The usual assumption is that the Higgs is in the broken phase $\mu^{2}>0$ from the beginning at the big bang. The EW phase transition is then taking place when the universe is cooling down below the critical temperature $T_{c}=\sqrt{\mu^{2} / g_{T}}$, meaning $g_{T} T^{2}-\mu^{2}<0$ when $T<T_{c}$. My analysis in contrast shows that above the phase transition point $\mu_{0}$ the $\mathrm{SM}$ is in the symmetric phase with $-\mu^{2} \rightarrow m_{0}^{2}=\left(m_{H}^{2}+\delta m_{H}^{2}\right) / 2>0$, and the EW phase transition is essentially triggered by the Higgs mechanism, at least it can happen only after the Higgs mechanism has taken place, thus $\mu_{\mathrm{EW}}<\mu_{\mathrm{HM}}=\mu_{0}$. The relevant question here is which of the terms $\delta m^{2}$ or $g_{T} T^{2}$ is leading in the relevant epoch of in early universe? I find $m_{0}^{2}\left(\mu=M_{\mathrm{Pl}}\right) \simeq$ $0.87 \times 10^{-3} M_{\mathrm{Pl}}^{2}$ such that $T\left(\mu=\mu_{0}\right) \simeq 1.62 \times 10^{29}{ }^{\circ} \mathrm{K}$ and $T\left(\mu=M_{\mathrm{Pl}}\right) \simeq 4.18 \times 10^{30}{ }^{\circ} \mathrm{K}$. We note that $T_{\mathrm{Pl}} \simeq 1.42 \times 10^{32}{ }^{\circ} \mathrm{K}$ (temperature of the Big Bang). The coefficient $g_{T}$ is given by $g_{T}=\frac{1}{4 v^{2}}\left(2 m_{W}^{2}+m_{Z}^{2}+2 m_{t}^{2}+\frac{1}{2} m_{H}^{2}\right)=\frac{1}{16}\left[3 g^{2}+g^{\prime 2}+4 y_{t}^{2}+\frac{2}{3} \lambda\right] \approx 0.0983 \sim 0.1$ using the results of Table 2 at scale $M_{\mathrm{Pl}}$. The dramatic jump in $m_{0}^{2}$ at $\mu_{0}$ in any case drags the Higgs into the broken phase not far below $\mu_{0}$ as illustrated in Fig. 3

\section{The Higgs hierarchy and its impact on inflation}

Cosmological inflation [35 41] requires an exponential growth of the Friedman-RobertsonWalker radius of the universe $a(t)$, i.e. $a(t) \propto \mathrm{e}^{H t}$ with $H(t)=\dot{a} / a(t)$ the Hubble constant at cosmic time $t$. $\dot{X}$ denotes the time derivative of $X$. Inflation is able to solve the flatness problem (why is the actual energy density of the universe so close to the critical density, the unique value which a flat universe must have as a limiting case between the closed and the open universes) and the horizon problem (without inflation what we seen when we look at the CMB radiation, we would see a huge patch which at the time of last scattering was outside the causal horizon, while the pattern is observed to be uniform over all sky). The inflation term comes in via the SM energy-momentum tensor and adds to the r.h.s. of the 

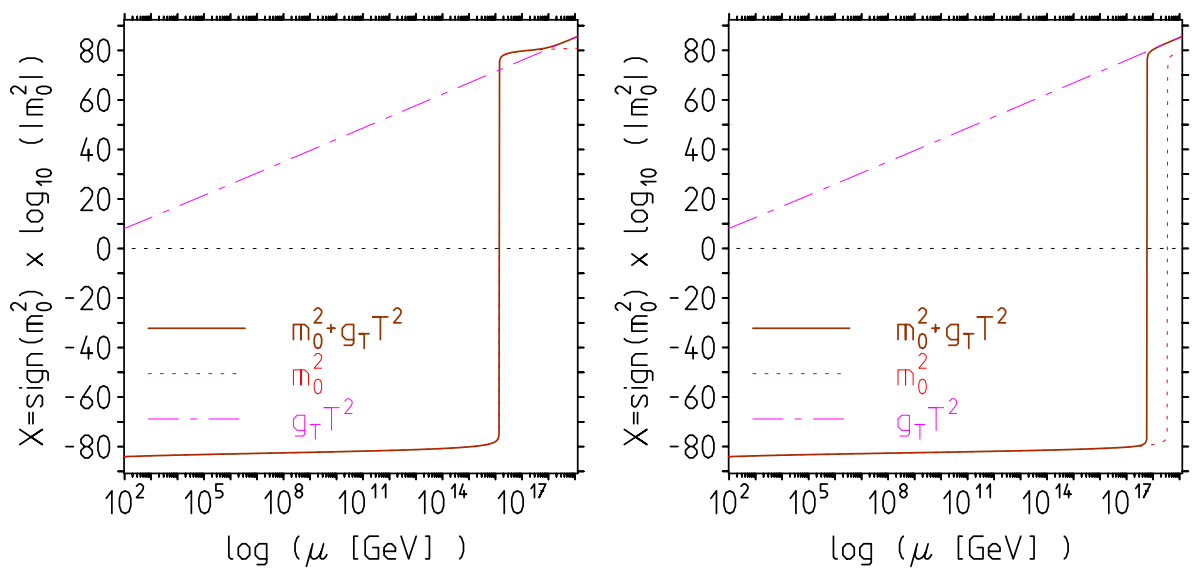

Figure 3: The role of the Higgs in the finite temperature SM. Left: for $\mu_{0} \sim 1.4 \times 10^{16} \mathrm{GeV}$ $\left(M_{H} \sim 126 \mathrm{GeV}, M_{t} \sim 173.5 \mathrm{GeV}\right)$. Right: finite temperature delayed transition for $\mu_{0} \sim 6 \times 10^{17} \mathrm{GeV}\left(M_{H} \sim 124 \mathrm{GeV}, M_{t} \sim 175 \mathrm{GeV}\right)$, the $m_{0}^{2}$ term alone is flipping at about $\mu_{0} \sim 3.5 \times 10^{18} \mathrm{GeV}$.

Friedmann equation

$$
\ell^{2}\left(V(\phi)+\frac{1}{2} \dot{\phi}^{2}\right)
$$

where $\ell^{2}=8 \pi G / 3, M_{\mathrm{Pl}}=(G)^{-1 / 2}$ is the Planck mass, $G$ Newton's gravitational constant.

In the SM the Higgs contribution to the energy-momentum tensor in terms of energy density and pressure amounts to

$$
\rho_{\phi}=\frac{1}{2} \dot{\phi}^{2}+V(\phi) ; \quad p_{\phi}=\frac{1}{2} \dot{\phi}^{2}-V(\phi) .
$$

The second Friedman equation $\ddot{a} / a=-\frac{\ell^{2}}{2}(\rho+3 p)$ tells us that the condition for growth $\ddot{a}>0$ requires $p<-\rho / 3$ and hence $\frac{1}{2} \dot{\phi}^{2}<V(\phi)$. CMB observations strongly favor the slow-roll inflation $\frac{1}{2} \dot{\phi}^{2} \ll V(\phi)$ condition. Indeed the Planck mission measured $w=p / \rho=$ $-1.13_{-0.10}^{+0.13}$. The first Friedman equation reads $\dot{a}^{2} / a^{2}+k / a^{2}=\ell^{2} \rho$ and may be written as $H^{2}=\ell^{2}\left[V(\phi)+\frac{1}{2} \dot{\phi}^{2}\right]=\ell^{2} \rho$, while the field equation reads $\ddot{\phi}+3 H \dot{\phi}=-V^{\prime}(\phi)=$ $-\mathrm{d} V(\phi) / \mathrm{d} \phi$. Note that the kinetic term $\dot{\phi}^{2}$ is controlled by $\dot{H}=-\frac{3}{2} \ell^{2} \dot{\phi}^{2}=\ell^{2} \rho(q-1)$, i.e. by the observationally controlled deceleration parameter $q(t)=-\ddot{a} a / \dot{a}^{2}$.

Inflation requires the presence of a dominating dark energy contribution, characterized by the equation of state $p / \rho=-1$. This is precisely what the SM in the symmetric phase suggests. Provided the Higgs potential remains stable ( $\lambda$ positive) a huge positive bare mass square at least naively supports the Gaussian slow-roll inflation condition. Since both $\lambda$ and $m^{2}$ for the first time are numerically fairly well known, quantitative conclusions concerning the phenomenologically established features of inflation should be possible solely on the basis of SM properties. In a phase where the mass term is dominating, the behavior is characterized 
by a free massive scalar field with potential $V=\frac{m^{2}}{2} \phi^{2}$ such that $H^{2}=(\dot{a} / a)^{2}=\frac{m^{2}}{6} \phi^{2}$ and $\ddot{\phi}+3 \dot{H}(\phi)=m^{2} \phi$ which is a harmonic oscillator with friction. It tells us that the Higgs field is decaying more or less rapidly, or looking back in time the Higgs field must grow exponentially implying that $\phi$ must have been very large in the early universe. This does not conflict with the expectation that the SM Higgs field at low energies is of moderate size, as it is renormalized by wave function renormalization factors which depend logarithmically only on the renormalization scale and thus on the cutoff. A huge Higgs field at early times in fact is crucial for SM inflation to work, otherwise we would not get a sufficient amount inflation. What also helps is the quartically enhanced cosmological constant (CC) provided by the SM Higgs. In Ref. [3] we have shown that the corresponding vacuum energy density is actually calculable by perturbative means, with the result

$$
V(0)=\frac{m^{2}}{2}\left\langle 0\left|\phi^{2}\right| 0\right\rangle+\frac{\lambda}{24}\left\langle 0\left|\phi^{4}\right| 0\right\rangle=\frac{m^{2}}{2} \Xi+\frac{\lambda}{8} \Xi^{2} ; \Xi=\frac{M_{\mathrm{Pl}}^{2}}{16 \pi^{2}} .
$$

With $m^{2} \approx \delta m^{2}=\frac{M_{\mathrm{Pl}}^{2}}{32 \pi^{2}} C(\mu)$ the vacuum energy density reads

$$
\rho_{\Lambda 0}=\rho_{\Lambda}+\frac{M_{\mathrm{Pl}}^{4}}{\left(16 \pi^{2}\right)^{2}} X(\mu)
$$

with $X(\mu)=\frac{1}{8}(2 C(\mu)+\lambda(\mu))$. Thus $X(\mu)=0$ close to the zero of $C(\mu)$, which takes relatively large negative values at lower energies (see Fig. 2) 2 . Thus, surprisingly, the cosmological constant and the Higgs mass term have strongly correlated matching points, where the renormalized low energy quantity coincides with the bare parameter and quadratic as well as quartic cutoff effects are nullified not far from each other near the EW phase transition point (see Ref. [3] for details). Below the corresponding zeros the renormalized parameter relations and parameter running applies and as low energy parameters there is no reason why the renormalized quantities cannot be small. As a result, the SM predicts a huge timedependent $\mathrm{CC}$, at $M_{\mathrm{Pl}}$ equivalent to $\rho_{\phi} \simeq V(\phi) \sim 2.77 M_{\mathrm{Pl}}^{4} \sim 6.13 \times 10^{76} \mathrm{GeV}^{4}$, for the initial field value $\phi_{i} \simeq 4.51 M_{\mathrm{Pl}}$ at Planck time $t_{i}=t_{\mathrm{Pl}}$, while the value observed today is $\rho_{\text {vac }}=\mu_{\Lambda}^{4}$ with $\mu_{\Lambda} \sim 0.002 \mathrm{eV}$ ! The short distance versus long distance "matching-patch" separates the regime where we look at the bare system form the "illusory world" we see at low energies where one has lost the memory oft the cutoff.

The SM inflation pattern is impressively supported by observation, most recently by the Planck 2013 results [42]. The cosmological constant is characterized by the equation of state $w=p / \rho=-1$, and in our scenario is a prediction of the SM for times before the phase transition when $\mu>\mu_{0}$. During the very early inflation era, when $\frac{1}{2} \dot{\phi}^{2} \ll V(\phi)$, the Higgs field is decaying exponentially and a large Higgs field at the Planck scale in not unnatural. In fact we need a huge field strength $\phi_{i} \simeq 4.51 M_{\mathrm{Pl}}$ at Planck time $t_{i}=t_{\mathrm{Pl}}$, in order to get an amount of inflation $N_{e}=\ln \left(a\left(t_{e}\right) / a\left(t_{i}\right)\right)=\int_{t_{i}}^{t_{e}} H(t) \mathrm{d} t>60$, which is required as a minimum in order to solve the CMB horizon problem. For the initial field value mentioned we obtain $N_{e} \approx 65$ and inflation ends at about $t_{e} \simeq 450 t_{\mathrm{Pl}}$ with $\phi_{e} \simeq 2 \times 10^{-3} M_{\mathrm{Pl}}$. Inflation in any case

\footnotetext{
${ }^{2}$ The non-vanishing $\left\langle 0\left|\phi^{2}\right| 0\right\rangle$ also implies a shift of the effective mass of the Higgs by $m^{\prime 2}=m^{2}+\frac{\lambda}{2} \Xi$.
} 
would be stopped by the phase transition when $\mu=\mu_{0}$, however, due to the exponential decay of the field inflation stops much earlier and field oscillations set in before the phase transition is reached. In our scenario, in the symmetric phase, the effective number of relativistic degrees of freedom is $g_{*}(T)=g_{B}(T)+\frac{7}{8} g_{f}(T)=102.75$ such that the Hubble constant, during the very early radiation dominated era, reads $H=\ell \sqrt{\rho} \simeq 1.66\left(k_{B} T\right)^{2} \sqrt{102.75} M_{\mathrm{Pl}}^{-1}$, or at Planck time $H_{i} \simeq 16.83 M_{\mathrm{Pl}} \approx 2.05 \times 10^{20} \mathrm{GeV} \simeq 3.12 \times 10^{44} \mathrm{sec}^{-1}$ as an initial value, which however decreases with $1 / T^{4}$, such that the pure inflation dark energy Hubble constant given by $H_{\phi} \simeq \ell \sqrt{V(\phi)} \approx 4.81 M_{\mathrm{Pl}} \approx 5.88 \times 10^{20} \mathrm{GeV} \simeq 8.93 \times 10^{43} \mathrm{sec}^{-1}$ becomes dominant and inflation sets in. While $\phi$ is large the interaction term of the Higgs Lagrangian will be dominating at first. As $\phi$ is decreasing the mass term will be dominating for some time before inflation stops.

\section{Remarks concerning reheating and baryogenesis}

The four Higgses near the Planck scale have an effective mass about $m_{H 0} \simeq 3.6 \times 10^{17} \mathrm{GeV}$ and thus can be produces in processes like $W W \rightarrow H H$ or $t \bar{t} \rightarrow H$ at times at and after the big bang. The big difference to standard big bang scenarios is that the Higgses are primordial, i.e. they exist as modes in the Planck medium in advance of being produced by high energy radiation processes. A Higgs in this phase has a width dominated by $H \rightarrow t \bar{t}$ decay, since direct couplings $H W W$ and $H Z Z$ are absent in the symmetric phase. One estimates

$$
\Gamma_{H} \simeq \frac{m_{H 0}}{16 \pi} N_{c} y_{t}^{2}\left(M_{\mathrm{Pl}}\right) \simeq 7.5 \times 10^{-3} m_{H 0} \simeq 2.7 \times 10^{15} \mathrm{GeV}
$$

yields a life time $\tau_{H}=1 / \Gamma_{H} \simeq 2.5 \times 10^{-40}$ sec. This is relatively large in terms of Planck times $t_{\mathrm{Pl}} \simeq 5.4 \times 10^{-44} \mathrm{sec}$. It supports the possibility that the coupling do not change immediately when dramatic cooling due to inflation takes place. The SM predicts that the Higgses produce top/anti-top quark radiation most abundantly. This means that reheating is mainly provided by $H \rightarrow t \bar{t}$ decays. In addition we estimate that $\Gamma_{H} \ll H(t)=\dot{a}(t) / a(t)$ during inflation, before the phase transition takes place. The energy density of top/anti-top quarks produced by the Higgs decays satisfies the conservation equation (see e.g. Ref. [43])

$$
\dot{\rho}_{t}+3 H\left(\rho_{t}+p_{t}\right)=\Gamma_{H} \rho_{\phi} .
$$

Since the top quarks are relativistic $p_{t}=\rho_{t} / 3$, and provided the energy density is still dominated by the inflaton, we can estimate the maximum top radiation density. As a result one obtains

$$
\begin{aligned}
\rho_{t \max } & \leq(3 / 8)^{8 / 5} t_{i} \Gamma_{H} \rho_{\phi}\left(t_{i}\right)=0.139\left(\Gamma_{H} / H\left(t_{i}\right)\right) \rho_{\phi}\left(t_{i}\right) \\
& \simeq 0.139 \frac{3 \sqrt{3} y_{t}^{2}\left(M_{\mathrm{Pl}}\right)}{64 \pi \sqrt{\pi}} M_{\mathrm{Pl}}^{3} m_{H 0} \simeq 1.6 \times 10^{71} \mathrm{GeV}^{4}
\end{aligned}
$$

with $t_{i}$ the Planck time. 
Concerning the possibility of baryogenesis, baryon-number violating interactions in the low energy effective SM (LEESM) scenario naturally are the close-by dimension 6 effective four-fermion interactions discussed first by Weinberg [44]. Usually, it is assumed that some unknown very heavy particle $X$ is responsible for baryogenesis. The first stage is characterized by $k_{B} T>m_{X}$ when we have thermal equilibrium and $X$ production and $X$ decay are in balance. The second stage follows if $H \approx \Gamma_{X}$ and $k_{B} T<m_{X}$ implying that $X$ production is suppressed and the system moves out of equilibrium. Our $X$ is the Higgs, with its known properties. Besides the predominant "would-be charged" Higgs decays $H^{+} \rightarrow t \bar{b}$ and $H^{-} \rightarrow b \bar{t}$, with rates proportional to $y_{t} y_{b}$, decays proportional to the $C P$-violating $\mathrm{CKM}$ matrix-elements $V_{t d}$ and $V_{u b} H^{+} \rightarrow t \bar{d}, u \bar{b}$ and $H^{-} \rightarrow b \bar{u}, d \bar{t}$ are important as a condition for baryogenesis. At inflation times we have $H^{+} \rightarrow t \bar{d}$ with rate $\propto y_{t} y_{d} V_{t d} \sim 5.5 \times 10^{-8}(1-\rho-\mathrm{i} \eta)$ and $H^{-} \rightarrow b \bar{u}$ with rate $\propto y_{b} y_{u} V_{u b} \sim 1.2 \times 10^{-9}(\rho-\mathrm{i} \eta)$, where $\rho=0.131, \eta=0.345$. The rates compare to the dominant $t$-mode 3 with relative rate $y_{t}^{2} \approx 0.123$. As mentioned before, matter production is preferably into fermion pairs with the biggest Yukawa couplings. After the EW phase transition the now heavy states decay into the lighter ones, with the smaller Yukawa couplings. Thus the major part of normal matter is produced via the heavy states which are cascading down the CKM coupling scheme. Apparently in such a scenario the system likely would intermittently be far from equilibrium while approaching the EW phase transition, and the dynamics behind could be important for the explanation of the baryon-asymmetry. So, likely in this scenario the origin of the baryon-asymmetry may have a different explanation than thought so far.

\section{Conclusion}

The main conclusions have been given in the abstract already. Here we would like to point out the importance of an extended analysis of the possible consequences of the SM physics. One of our main assumptions has been the one that physics beyond the SM is not needed to understand the early universe. The point is that in the LEESM scenario unseen physics can naturally be expected, however, it must be natural in the sense of a low energy expansion. Grand unified theories as well as a supersymmetrized SM are not natural, because they require an improbably high amount of conspiracy of very many modes, while the emergence of an extra $U(1)$ or a $S U(4)$ look much more natural. What is also ruled out are additional fermion families. They definitely would spoil the present interplay of couplings which make the extrapolation up to the Planck scale working.

We once more point out that there is no hierarchy problem in the broken phase of the SM. All particle masses, the ones protected by symmetries as well as the unprotected Higgs, are proportional to the Higgs vacuum expectation value times a coupling which is subject to logarithmic scale dependence only. The Higgs VEV is an order parameter determined by collective long range properties of the system. If $v$ would be of order $M_{\mathrm{Pl}}$ the notion of spontaneous symmetry breaking would be obsolete, since the symmetry would not be

\footnotetext{
${ }^{3}$ The next-to-leading $b$-quark rates are reduced by the branching fraction $4.4 \times 10^{-4}$ and the $\tau$-lepton rates are lower by $2.2 \times 10^{-4}$.
} 
recovered at the high scale. We should remember that the UV structure is the same in the symmetric and in the broken phase, there cannot be any additional UV cutoff sensitivity generated by the Higgs VEV. That $v$ is much smaller than the cutoff, in principle can be checked by putting the SM on a lattice of lattice spacing $a$ and then calculate $v a$, which should turn out to be extremely small $O\left(10^{-16}\right)$, which is possible if the temperature turns out to be very close just below the critical temperature. This again is expected to be the result of the specific conspiracy of the various couplings of the SM.

In any case, a super symmetric or any other extension of the SM cannot be motivated by the (non-existing) hierarchy problem. The Higgs in a supersymmetric extension of the SM cannot be the inflaton and provide the necessary dark energy feeding inflation.

How do we get cold dark matter? If the right-handed sterile singlet neutrinos are Majorana particles exhibiting naturally a large Majorana mass term not protected by any symmetry and not participating in the Higgs spontaneous symmetry breaking could play a role here. Such sterile Majorana neutrinos would naturally have masses of the order of the Planck scale and therefore not affect the running parameters of the SM, as they do not couple directly to any of the SM fields and as they satisfy the decoupling theorem. At the same time they would provide the seasaw mechanism which would explain the smallness of the neutrino masses. But also an extra hidden $S U(4)$ could play a role here, by forming stable bosonic quartet bound states. Cold dark matter could be dominated by bound energy, similarly to the case of normal baryonic matter with respect to QCD. Since $S U(4)$ bound states are bosonic formation of structures and distribution of corresponding dark matter would be very different form that of normal fermionic matter.

We also note that new physics like the existence of axions which could play a key role in the issues of the strong CP problem, have a natural place in a renormalizable low energy effective world.

As we have seen, a big issue is the very delicate conspiracy between SM couplings. Therefore precision determinations of parameters are more important than ever and a real challenge for experiments at the LHC and at a future ILC, which may improve substantially $\lambda, y_{t}$ and $\alpha_{s}$. But also low energy hadron facilities have to play an important role as needed for a better control of the non-perturbative hadronic effects in $\alpha\left(M_{Z}\right)$ and $\alpha_{2}\left(M_{Z}\right)$. It is important to note that, provided there is essentially no other stuff, coming closer to the properties of the Planck ether is only possible by pushing high precision physics. Thus higher order calculations and high precision determinations of parameters are of paramount importance. Note that the precise value of the top Yukawa contribution plays a particular role for the precise location of the zero of the coefficient of the quadratic divergence, as it is enhanced by a factor 6 relative to the Higgs self-coupling. Whether the Higgs is the inflaton with the right properties depends crucially on the precise point in the $\left(\lambda, y_{t}\right)$-plane. The window for this is very narrow.

Our analysis shows that the role of the Higgs is not just to provide masses to SM particles, it also plays a key role in cosmology: for some time at and after the big bang the Higgs is the only particle which directly talks to gravity and directly takes part in the evolution of the universe. It is the only SM particle which directly talks to the vacuum in the early universe. Later, in the low energy phase, contributions from the EW phase transition through the 
Higgs VEV and from the QCD phase transition through quark and gluon condensates come into play. This has to be investigated yet. Nevertheless, the Higgs very likely is the object able to provide negative pressure and likely is responsible for blowing continuously energy into the expanding universe according to the established dark energy which still is existing today. While the Higgs likely was playing a dominating role in shaping the early universe, in our present world the Higgs hides itself so much that it took decades to actually find it, after theorists had proposed it as being the source of the masses of the SM particles.

\section{Acknowledgments}

Thanks to the organizers for the invitation and support to the XX Epiphany Conference, and for giving me the opportunity to present this talk.

\section{References}

[1] F. Jegerlehner, Acta Phys. Polon. B 45 (2014) 1167; arXiv:1304.7813 [hep-ph].

[2] F. Jegerlehner, The hierarchy problem of the electroweak Standard Model revisited, arXiv:1305.6652 [hep-ph].

[3] F. Jegerlehner, Acta Phys. Polon. B 45 (2014) 1215; arXiv:1402.3738 [hep-ph].

[4] G. Aad et al. [ATLAS Collaboration], Phys. Lett. B 716 (2012) 1; Science 338 (2012) 1576 .

[5] S. Chatrchyan et al. [CMS Collaboration], Phys. Lett. B 716 (2012) 30; Science 338 (2012) 1569.

[6] T. Hambye, K. Riesselmann, Phys. Rev. D 55 (1997) 7255.

[7] F. Jegerlehner, Phys. Rev. D 16 (1977) 397.

[8] F. Jegerlehner, The Vector Boson and Graviton Propagators in the Presence of Multipole Forces, Helv. Phys. Acta 51 (1978) 783.

[9] M. Holthausen, K. S. Lim, M. Lindner, JHEP 1202 (2012) 037.

[10] L. N. Mihaila, J. Salomon, M. Steinhauser, Phys. Rev. Lett. 108 (2012) 151602.

[11] K. G. Chetyrkin, M. F. Zoller, JHEP 1206 (2012) 033.

[12] A. V. Bednyakov, A. F. Pikelner, V. N. Velizhanin, arXiv:1210.6873 [hep-ph];

[13] A. V. Bednyakov, A. F. Pikelner, V. N. Velizhanin, arXiv:1212.6829 [hep-ph].

[14] K. G. Chetyrkin, M. F. Zoller, JHEP 1304 (2013) 091.

[15] A. V. Bednyakov, A. F. Pikelner, V. N. Velizhanin, arXiv:1303.4364 [hep-ph]. 
[16] F. Bezrukov, M.Yu. Kalmykov, B.A. Kniehl, M. Shaposhnikov, JHEP 1210 (2012) 140.

[17] G. Degrassi et al., JHEP 1208 (2012) 098,

[18] I. Masina, arXiv:1209.0393 [hep-ph].

[19] Y. Hamada, H. Kawai, K.Y. Oda, Phys. Rev. D 87 (2013) 053009.

[20] D. Buttazzo et al. arXiv:1307.3536 [hep-ph].

[21] J. Beringer et al. [Particle Data Group Collaboration], Review of Particle Physics (RPP), Phys. Rev. D 86 (2012) 010001.

[22] A. Juste, S. Mantry, A. Mitov, A. Penin, P. Skands, E. Varnes, M. Vos, S. Wimpenny, Determination of the top quark mass circa 2013: methods, subtleties, perspectives, arXiv:1310.0799 [hep-ph].

[23] M. J. G. Veltman, Acta Phys. Polon. B 12 (1981) 437.

[24] F. Jegerlehner, M.Yu. Kalmykov, B.A. Kniehl, Phys. Lett. B 722 (2013) 123.

[25] J. Fleischer, F. Jegerlehner, Phys. Rev. D 23 (1981) 2001.

[26] T. Appelquist, J. Carazzone, Phys. Rev. D 11 (1975) 2856.

[27] M. S. Al-sarhi, I. Jack, D. R. T. Jones, Z. Phys. C 55 (1992) 283.

[28] D. R. T. Jones, Phys. Rev. D 88 (2013) 098301.

[29] F. Jegerlehner, M. Y. .Kalmykov, O. Veretin, Nucl. Phys. B 641 (2002) 285; Nucl. Phys. B 658 (2003) 49.

[30] F. Jegerlehner, M. Y. .Kalmykov, Nucl. Phys. B 676 (2004) 365; Acta Phys. Polon. B 34 (2003) 5335.

[31] M. Dine, R. G. Leigh, P. Y. Huet, A. D. Linde, D. A. Linde, Phys. Rev. D 46 (1992) 550.

[32] D.A. Kirzhnits, JETP Lett. 15 (1972) 529; D.A. Kirzhnits, A.D. Linde, Phys. Lett. 72B (1972) 471.

[33] S. Weinberg, Phys. Rev. D9 (1974) 3357; L. Dolan, R. Jackiw, Phys. Rev. D9 (1974) 3320; D.A. Kirzhnits, A.D. Linde, JETP 40 (1974) 628.

[34] D.A. Kirzhnits, A.D. Linde, Ann. Phys. 101 (1976) 195.

[35] A. H. Guth, Phys. Rev. D 23 (1981) 347.

[36] A. A. Starobinsky, Phys. Lett. B 91 (1980) 99. 
[37] A. D. Linde, Phys. Lett. B 108 (1982) 389; Phys. Lett. B 129 (1983) 177.

[38] A. Albrecht, P. J. Steinhardt, Phys. Rev. Lett. 48 (1982) 1220.

[39] V. F. Mukhanov, G. V. Chibisov, JETP Lett. 33 (1981) 532 [Pisma Zh. Eksp. Teor. Fiz. 33 (1981) 549];

[40] V. F. Mukhanov, JETP Lett. 41 (1985) 493 [Pisma Zh. Eksp. Teor. Fiz. 41 (1985) 402];

[41] V. F. Mukhanov, H. A. Feldman, R. H. Brandenberger, Phys. Rept. 215 (1992) 203.

[42] P. A. R. Ade et al. [Planck Collab.], arXiv:1303.5062; arXiv:1303.5082; arXiv:1303.5084 [astro-ph.CO].

[43] S. Weinberg, Cosmology, Oxford, UK: Oxford Univ. Pr. (2008) 593 p

[44] S. Weinberg, Phys. Rev. Lett. 43 (1979) 1566. 